\title{
ESTUDIO DE ALGUNOS PROBLEMAS DE PANDEO DESDE UN ENFOQUE ESTOCÁSTICO
}

\section{(STUDY OF SOME BUCKLING PROBLEMS FORAM A STOCHASTIC APPROACH)}

\author{
José L. Romero Martín, Dr. Ingeniero de Caminos, Profesor Fac. Informática. \\ Emilio de la Rosa Oliver, Dr. Ingeniero de Caminos, Catedrático E.T.S.I.C.C. y P. \\ Universidad Politécnica de Madrid. España
}

Fecha de recepción: 11-V-90 406-5

\section{RESUMEN}

En este articulo, se exponen las diversas causas que justifican el interés de un análisis estocástico de los problemas de pandeo. El desarrollo efectuado se centra fundamentalmente en la aplicación del método de discretización, el cual ha sido descrito en un articulo previo (13). Como núcleo del mismo, se estudia el pandeo de una pieza recta con vínculos de tipo elástico, cuyos valores son considerados como variables aleatorias. Asimismo, se expone como ejemplo el caso concreto de una pieza con rigidez constante, aplicando el método de discretización en la resolución de la correspondiente ecuación trascendente estocástica. Finalmente, se indican otras aplicaciones del citado método, a la resolución de problemas de pandeo estocástico, tales como los de rigidez estocástica e imperfección inicial estocástica.

\section{SUMMARY}

In this paper, different motivations that justify the interest of stochastic analysis of buckling problems are presented. The work done is based mainly in the application of a discretization method described on a previous article (13). The main part of the work consists of the study of the buckling of a bar with elastic constraints, which are considered random variables. Likewise, for the concrete example of a bar with constant flexural rigidity, the stochastic trascendent equation is solved using the mentioned discretization method. Finally, other aplications of the method to the resolution of stochastic buckling problems, such as the stochastic flexural rigidity and stochastic initial imperfection are pointed out.

\section{INTRODUCCIÓN}

Históricamente, los estudios sobre la determinación de la carga de pandeo de una barra recta se remontan al siglo XVIII siendo Leonhar Euler el primero que resolvió de manera teórica dicho problema en 1744, obteniendo que el valor de dicha carga era inversamente proporcional al cuadrado de la longitud de la barra, lo cual al mismo tiempo era acorde con los resultados experimentales obtenidos por Musschenbroek treinta años antes. (v. Timoshenko [16] y Brush y Almorth [5]).

No obstante fueron necesarios muchos experimentos para confirmar de manera definitiva los resultados de Euler, debiéndose destacar en relación con esto los trabajos de Christie (1884), Considere (1889) y Tetmajer (1890 y 1896), los cuales pusieron de manifiesto que los valores de la tensión crítica $\sigma_{c r}$ se sitúan sobre la curva de Euler $\sigma_{\mathrm{cr}}=\pi^{2} \mathrm{E} / \lambda^{2}$ siempre que la esbeltez mecánica $\lambda$ (longitud/radio de giro) de la pieza sea tal que el pandeo ocurra para una tensión compresora inferior al límite de proporcionalidad $\sigma_{\mathrm{p}}$ del material (v. Timoshenko [16]).
Asimismo, con posterioridad a los trabajos de los citados autores, se ha investigado sobre el tema tanto en aspectos teóricos como experimentales, siendo quizá ésta una de las áreas del Cálculo de Estrủucturas que más publicaciones y comunicaciones ha suscitado.

Muchos de estos estudios han tratado y tratan de resolver las dificultades con las que tropieza la teoria de Euler cuando es aplicada a situaciones no ideales que son precisamente las que se presentan en la práctica. En este sentido pueden destacarse los siguientes factores:

1) Excentricidad desconocida en la aplicación de la carga

2) Desviación inicial de la forma recta

3) Pequeñas variaciones en las áreas y/o en la forma de secciones transversales

4) Articulaciones y empotramientos no perfectos

5) Defecto de homogeneidad del material,

ya que todos y cada uno de ellos modifican de alguna manera la carga de pandeo. Además factores como por 
ejemplo la imperfección inicial en la forma o las condiciones de vínculo varían de una estructura a otra. construida o fabricada en condiciones similares. Análogamente otros factores como el módulo de elasticidad, momento de inercia, etc. pueden variar dentro de una misma estructura. Debido a estas causas por un lado, y por otro a la incertidumbre que se tiene en el conocimiento de los citados factores, el valor de la carga de pandeo resulta impredecible.

Estas circunstancias han motivado el empleo de modelos estocásticos como técnica idónea para el estudio de estos problemas. Los métodos que se aplican para su resolución tratan de establecer correspondencias entre ciertos parámetros estadísticos relativos a la carga de pandeo con otros asociados a una descripción adecuada de las imperfecciones.

Para precisar todo lo anterior, consideremos el siguiente problema de pandeo estocástico de una pieza recta de longitud $L$, con vínculos elásticos en los extremos, de modo que las constantes $K_{1}, K_{2}$ y $K_{3}$ que definen dichos vínculos sean variables aleatorias y la rigidez EI sea un proceso estocástico de parámetro $x$ debido a pequeñas variaciones aleatorias del momento de inercia y/o del módulo de elasticidad (v. Fig. 1.1.)

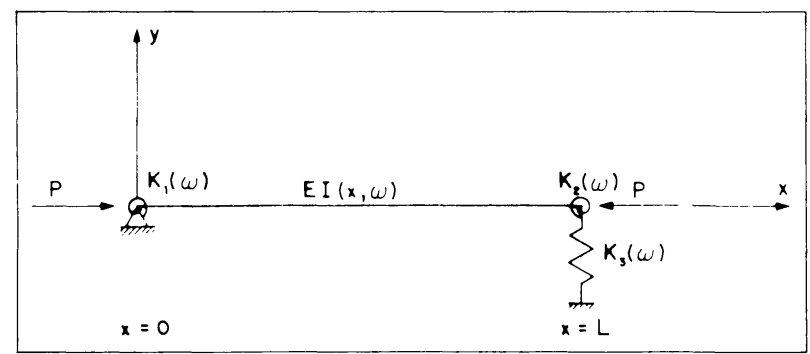

Fig. 1.1

En este caso, la carga de pandeo será una variable aleatoria $\mathrm{P}_{\mathrm{cr}}(\omega)$, la cual se corresponde con el autovalor positivo más pequeño del siguiente problema de contorno estocástico homogéneo: de la función de distribución de dicha variable aleato. ria. De esta manera se podrá obtener cierta información sobre la probabilidad de colapso para distintos valores de la carga de servicio $Q$ :

$\operatorname{Prob}\left\{P_{c r}(\omega) \leq Q\right\}=F_{P_{c r}}(Q)$

Por otro lado, los problemas de imperfección en la forma responden a un planteamiento semajante, pero dando lugar a problemas de contorno estocástico no homogéneos.

Para precisar esto, consideremos el caso de una pieza biarticulada de longitud $L$, ligeramente desviada de la forma rectilínea, de tal modo que dicha desviación puede ser modelizada por el proceso estocástico de parámetro $\mathrm{x}, \mathrm{y}^{*}(\mathrm{x}, \omega)$. Así para cada valor $\mathrm{P}$ distinto de los autovalores del problema homogéneo asociado, habrá solución distinta de la trivial y ésta será estocástica $Y(x, \omega)(v$. Fig. 1.2.)

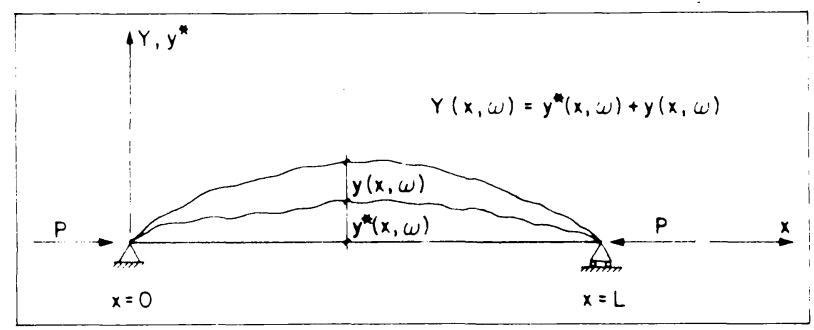

Fig. 1.2

El proceso $Y(x, \omega)=y^{*}(x, \omega)+y(x, \omega)$ se obtiene a partir de la solución del siguiente problema de contorno no homogéneo expresado en términos del desplazamiento adicional $\mathrm{y}(\mathrm{x}, \omega)$

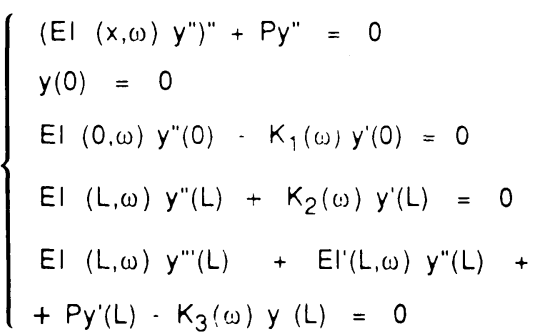

El interés en el análisis de problemas como el anterior, radica en obtener algunos momentos de $\mathrm{P}_{\mathrm{cr}}(\omega)$ tales como la media y la varianza e incluso aproximaciones
$\left\{\begin{array}{l}\left(E \mid(x) y^{\prime \prime}\right)+P y^{\prime \prime}=-P y^{* \prime}(x, \omega) \geq \\ y(0)=0 \\ y^{\prime \prime}(0)=0 \\ y(L)=0 \\ y^{\prime \prime}(L)=0\end{array}\right.$

El propósito en estos casos será encontrar relaciones entre los valores de la carga $P$ y la media y varianza del desplazamiento total $Y$ de la directriz en un punto $x=$ $\xi$ dado (habitualmente $\xi=L / 2$ ), o bien calcular la probabilidad de que el desplazamiento total en $x=\xi$ sea inferior a un valor preasignado, para un valor de $\mathrm{P}$ dado.

Los objetivos planteados en los problemas anterior mente expuestos se corresponden en cierto modo con los distintos criterios de tipo deterministas que se utilizan en la práctica para el cálculo de la carga de colapso de la pieza, según se trate de problemas 
homogéneos (de autovalores) o no homogéneos. Este modo de proceder enfocando el problema estocásticamente, permite introducir de una manera directa el concepto de fiabilidad, a partir de la probabilidad de ruina o colapso.

Como referencias bibliográficas relacionadas con el estudio del pandeo estocástico, cabe destacar las publicaciones de Amazigo [1], Boyce [2], Bernard y Bogdanoff [3], Budiansky y Hutchinson [4], Fraser [6], Fraser y Budiansky [7], Jacquot [9], Perry [10] y Roorda [11], los cuales tratan fundamentalmente el problema de imperfección en la forma y desde un enfoque eminentemente teórico, excepto el trabajo de Perry que es de carácter marcadamente experimental.

Por nuestra parte, en el presente artículo, realizamos el estudio del pandeo de una pieza recta con vínculos elásticos cuyas constantes son consideradas como variables aleatorias. Este análisis permite valorar la influencia de la aleatoriedad de las condiciones de vínculo en la aleatoriedad de la carga de pandeo, lo que consideramos de gran interés para las aplicaciones.

Dicho estudio se ha abordado mediante la aplicación de dos procedimientos distintos: perturbación y discretización. Se destaca la ventaja que supone la aplicación a este caso, del segundo procedimiento frente al primero. Asimismo, se expone como ejemplo, el caso concreto de una pieza con rigidez constante, aplicando el método de discretización en la resolución de la correspondiente ecuación trascendente estocástica.

Por último, se indican otras aplicaciones del método de discretización, en la resolución de problemas de pandeo estocástico, tales como los de rigidez estocástica e imperfección inicial estocástica.

El citado método de discretización ha sido expuesto en un número anterior de esta revista [13], pudiendo considerarse este artículo como una segunda parte de aquél en lo que a las aplicaciones del método se refiere.

\section{PANDEO DE UNA PIEZA RECTA CON VÍNCULOS DE TIPO ELÁSTICO ALEATORIOS}

Es frecuente en la práctica de la construcción, considerar como articuladas piezas que puedan tener un empotramiento parcial, pero del cual se desconoce el grado de empotramiento. En estos casos la hipótesis nos deja del lado de la seguridad. También hay situaciones, donde se considera a la pieza perfectamente empotrada en sus extremos, pudiendo ser sin embargo la realidad muy distinta, debido a pequeños defectos constructivos.

(c) Consejo Superior de Investigaciones Científicas

Licencia Creative Commons 3.0 España (by-nc)
Por otro lado las normas consideran también aquellos casos de piezas empotradas elásticamente en estructuras constituidas por vigas y pilares, definiendo las constantes de empotramiento elástico de cada nudo a partir de las rigideces y longitudes de los elementos (vigas y pilares) que concurren en el mismo. La determinación experimental del valor de estas constantes en un gran número de estructuras iguales (construidas en condiciones similares) pondría de manifiesto la presencia de una cierta dispersión.

Precisamente y en relación con lo expuesto, el objetivo de este apartado es estudiar la influencia de la aleatoriedad de las condiciones de vínculo en la aleatoriedad de la carga de pandeo.

Para este análisis se ha supuesto el caso de una pieza recta con rigidez $\mathrm{El}(\mathrm{x})$ (determinista) con empotramiento elástico en su extremo izquierdo y empotramiento elástico y apoyo elástico en el derecho (v. Fig. 2.1).

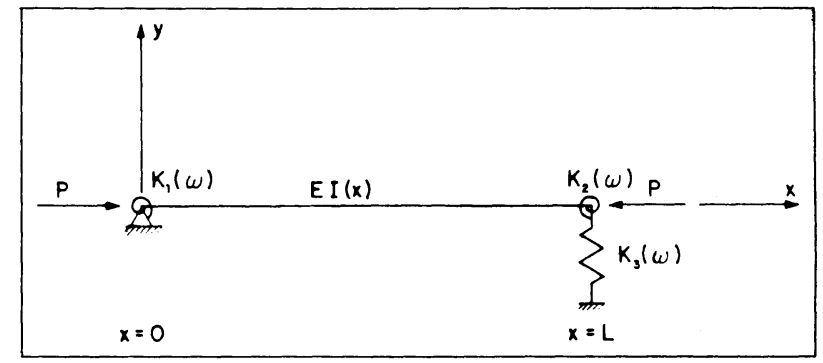

Fig. 2.1

Asimismo las variables aleatorias que definen las constantes de los empotramientos y del apoyo elástico, se han considerado independientes.

La carga de pandeo $P_{c r}(\omega)$ para este caso equivale al autovalor positivo más pequeño del siguiente problema de autovalores estocásticos:

בֶ.

$$
\begin{aligned}
& \left(E I(x) y^{\prime \prime}\right)^{\prime \prime}+P y^{\prime \prime}=0 \\
& y(0)=0 \\
& E I(0) y^{\prime \prime}(0)-K_{1}(\omega) y^{\prime}(0)=0 \\
& E I(L) y^{\prime \prime}(L)+K_{2}(\omega) y^{\prime}(L)=0 \\
& E I(L) y^{\prime \prime \prime}(L)+E I^{\prime}(L) y^{\prime \prime}(L)+P y^{\prime}(L)-K_{3}(\omega) y(L)=0
\end{aligned}
$$

El análisis estocástico de este problema puede abordarse a partir de métodos tales como los de Taylor, Monte Carlo, discretización, etc. En concreto se determinan aquí los sucesivos problemas deterministas que resultan respectivamente de aplicar el método Taylor mediante la técnica de perturbación y el de discretización al problema estocástico (2.1) (v. [12]).

http://informesdelaconstruccion.revistas.csic.es 


\section{Perturbación:}

$$
\begin{aligned}
\text { Haciendo } & y(x) & =\sum_{i, j, k \geq 0} y_{i j k}(x) u^{i} v^{j} w^{k} \quad y \\
\text { donde } & P & =\sum_{i, j, k \geq 0} P_{i j k} u^{i} v^{j} w^{k} \\
\text { con } & u & =k_{1} \cdot \bar{K}_{1} \quad v=k_{2} .
\end{aligned}
$$

\section{sustituyendo en (2.1) se obtiene:}

$\bar{K}_{2}, w=K_{3}-\bar{K}_{3}$, con $\bar{K}_{i}=E\left[K_{i}\right] ; i=1,2,3$

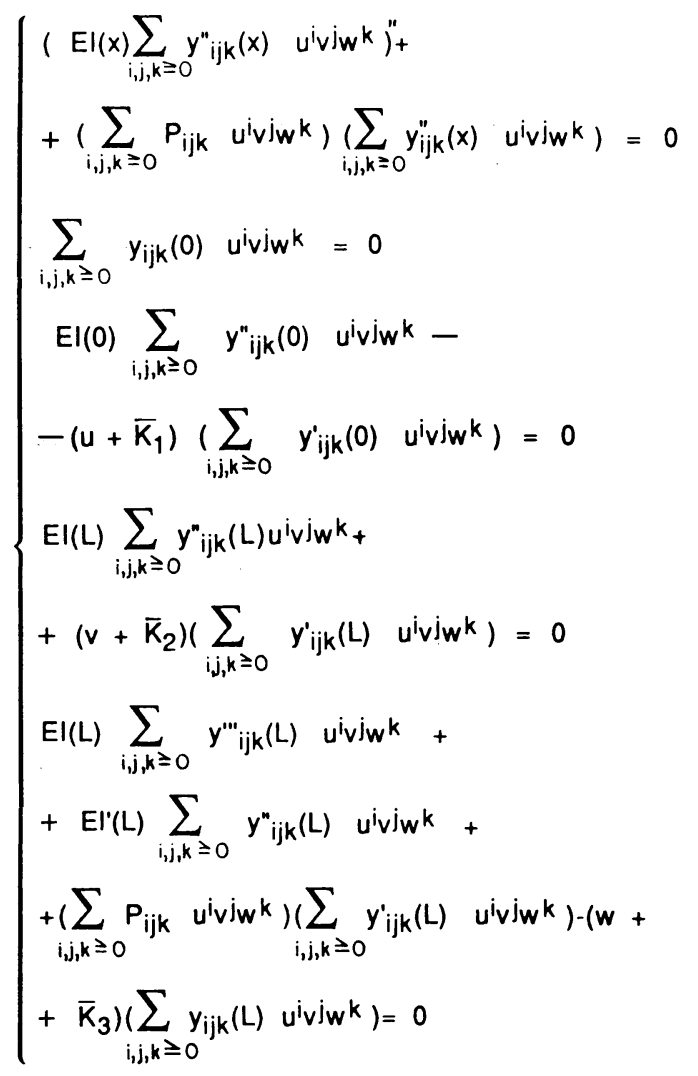

Añadiendo ahora una condición de normalización como

$$
y^{\prime \prime \prime}(0)=1 \equiv \sum_{i, j, k \geq 0} y^{\prime \prime \prime i j k} \quad(0) u^{i} v^{j} w_{w k}=1
$$

queda fijada una autofunción $\mathrm{y}(\mathrm{x})$ del subespacio asociado al autovalor $P_{c r}$. Puede demostrarse que esta condición no afecta a la determinación de los valores $P_{i j k} ; i, j, k \geq 0$ (v. Haines [8]).

Anulando ahora cada uno de los coeficientes de las potencias uiviwk; i,j, $k \geq 0$ en cada una de las ecuaciones de (2.2) y en (2.3) se obtiene:
Obsérvese que (2.4) es el problema determinista que resulta de sustituir en (2.1) $\mathrm{K}_{1}, \mathrm{~K}_{2}$ y $\mathrm{K}_{3}$ por sus respectivos valores medios

$$
\bar{K}_{i}=E\left[K_{i}\right] ; \quad i=1,2,3 .
$$

Anulando ahora los coeficientes de $u^{\circ} v^{0} w^{1}, u^{0} v^{1} w^{0} y$ $u^{1} v^{0} w^{0}$ se obtiene respectivamente:

$$
\left\{\begin{array}{l}
\left(E I(x) y_{001}^{\prime \prime}(x)\right)^{\prime \prime}+P_{000} y^{\prime \prime} 001(x)+ \\
+P_{001} y^{\prime \prime} 000(x)=0 \\
y_{001}(0)=0 \\
E I(0) y_{001}^{\prime \prime}(0)-\bar{K}_{1} y_{001}^{\prime}(0)=0 \\
E I(L) y_{001}^{\prime \prime}(L)+\bar{K}_{2} y_{001}^{\prime}(L)=0 \\
E I(L) y^{\prime \prime \prime} 001(L)+E^{\prime}(L) y_{001}^{\prime \prime}(L)+ \\
+P_{000} y_{001}^{\prime}(L)+P_{001} y_{000}^{\prime}(L) \\
\bar{K}_{3} y_{001}(L)-y_{000}(L)=0 \\
y^{\prime \prime \prime} 001(0)=0
\end{array}\right.
$$




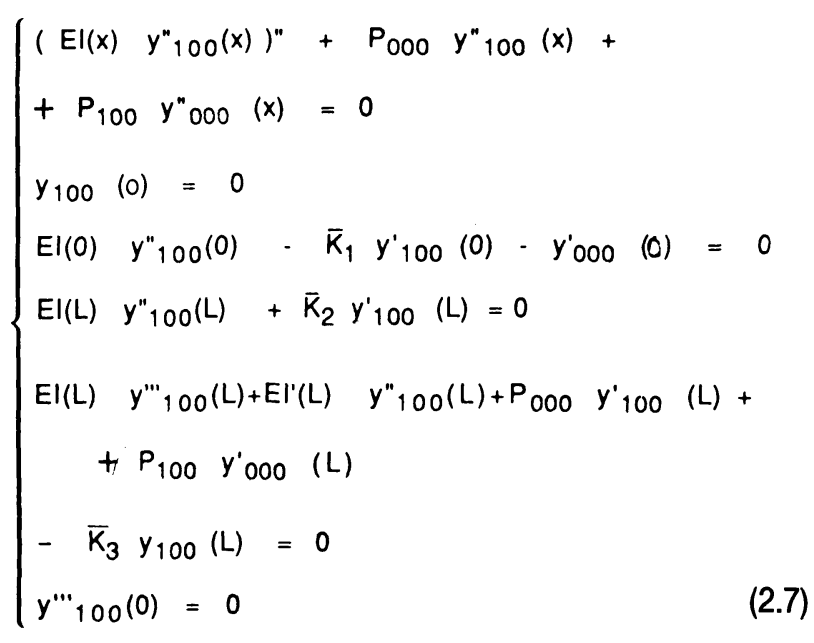

Análogamente, pueden obtenerse nuevas ecuaciones, anulando sucesivamente los coeficientes de uiviwk para $i+j+k \geq 2$.

La resolución de los sucesivos problemas (2.4), (2.5), (2.6), (2.7), etc. proporcionará las funciones $\mathrm{y}_{\mathrm{ijk}}(\mathrm{x}) ; \mathrm{i}, \mathrm{j}, \mathrm{k}$ $\geq 0$

$$
\text { ; } P_{i j k} ; i, j, k \geq 0
$$

y los coeficientes $P_{i j k} ; i, j, k \geq 0$.

El valor medio de la carga de pandeo será:

$$
\begin{aligned}
& E\left[P_{c r}\right]= \\
& =E\left[P_{000}+P_{001} w+P_{010^{2}}+P_{100} u+\sum_{i+j+k \geq 2} P_{i j k} u^{j} v^{j} u^{k}\right]= \\
& =P_{000}+\sum_{i+j+k \geq 2} P_{i j k} \quad E\left[u^{i}\right] E[v j] E\left[w^{k}\right]
\end{aligned}
$$

Por otro lado el momento de segundo orden para dicha carga es:

$E\left[P^{2} c r\right]=\sum_{\substack{i, j, k \geqslant 0 \\ \alpha, \beta \gamma}} P_{i j k} P_{\alpha \beta \gamma} E\left[u^{i+\alpha}\right] E\left[v^{j+\beta}\right] E\left[w^{k+\eta}=\right.$ $=P^{2} 000+P^{2} 10 D^{2}\left[K_{1}\right]+P^{2} 010 D^{2}\left[K_{2}\right]+P^{2} 001 D^{2}\left[K_{3}\right]+$

$+2 P_{000} \sum_{i, j, j \geq 2} P_{i j k} E\left[u^{i}\right] E\left[v^{j}\right] E\left[w^{k}\right]+$

$+2 \sum_{i+j+k \geq 2} P_{i j k}\left(P_{001} E\left[u^{j}\right] E[v]\right] E\left[w^{k+1}\right]+$

$\left.+P_{010} E\left[u^{i}\right] E\left[v^{j+1}\right] E\left[w^{k}\right]+P_{100} E\left[u^{i+1}\right] E[v j] E\left[w^{k}\right]\right)+$

$+\sum_{\substack{1+j+k \geqq 2 \\ \alpha+\beta+\gamma \geqslant 2}} P_{i j k} P_{\alpha \beta \gamma} E\left[u^{i+\alpha}\right] E\left[v^{j+\beta}\right] E\left[w^{k+\eta}\right.$
Las expresiones (2.8) y (2.9) permiten dar a partir de la resolución de los cuatro problemas (2.4) (2.5) (2.6) y (2.7) las siguientes estimaciones para la media y la varianza de $P_{c r}$ :

$$
\begin{aligned}
& E\left[P_{c r}\right] \equiv P_{000} \\
& D^{2}\left[P_{c r}\right] \cong P^{2} 100 D^{2}\left[K_{1}\right]+P^{2} 010 D^{2}\left[K_{2}\right]+ \\
& +P^{2} 001 D^{2}\left[K_{3}\right]
\end{aligned}
$$

\section{Discretización}

Sean

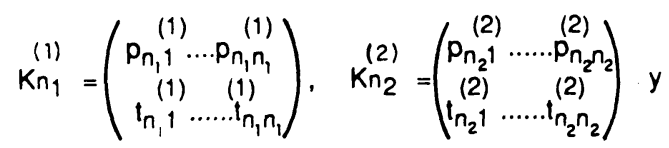

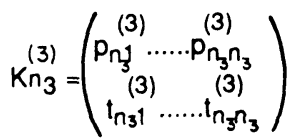

variables aleatorias discretas definidas como en el apartado 3 de [13] asociadas a las variables aleatorias $\mathrm{K}_{1}, \mathrm{~K}_{2}$ y $\mathrm{K}_{3}$ respectivamente. Así al problema (2.1) podremos asociarle este otro problema tambien estocástico
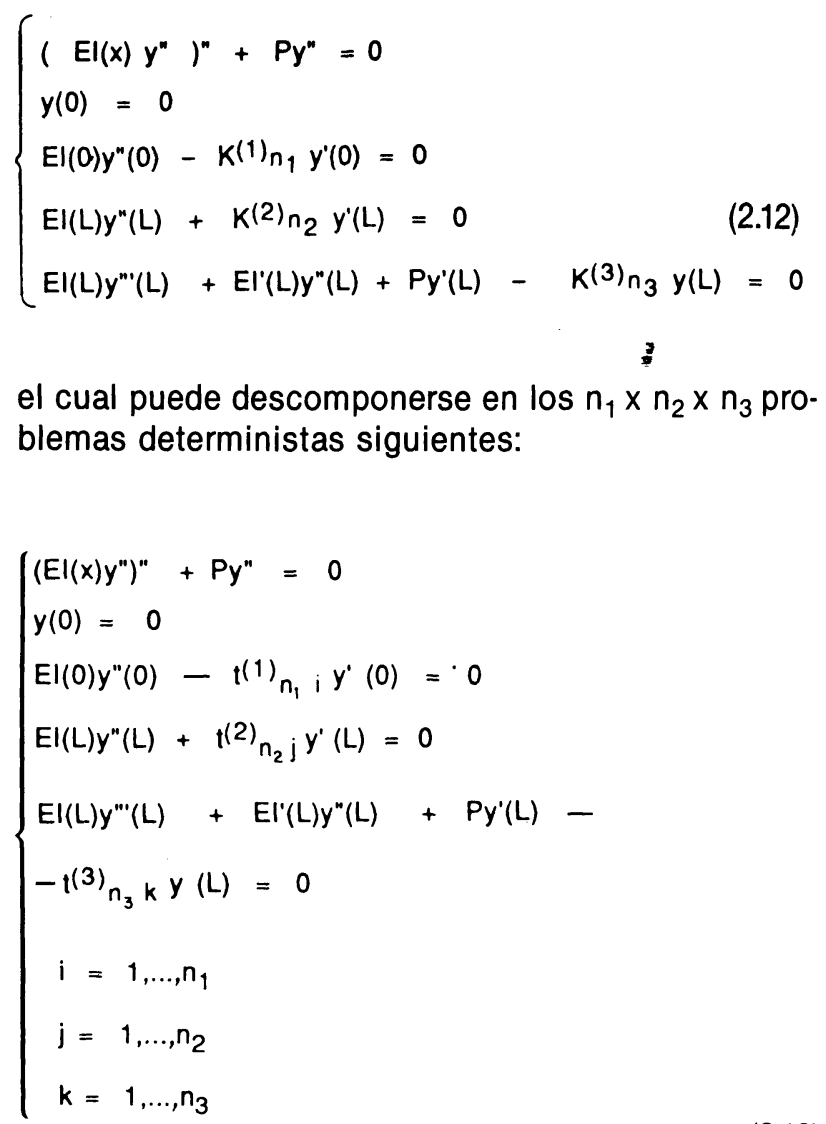
cada uno de los cuales lleva asociada la probabilidad

$$
P(1)_{n_{1}} ; P^{(2)} n_{n_{2} j} P(3) n_{n_{3}} k
$$

Así si llamamos g a la función que expresa la carga de pandeo $\mathrm{P}_{\mathrm{cr}}$ en términos de $\mathrm{K}_{1}, \mathrm{~K}_{2}, \mathrm{~K}_{3}$ a través del propio problema de autovalores (2.1), podremos poner

$$
\left(P_{c r}\right) n_{1} n_{2} n_{3}=g\left(K^{(1)} n_{1}, K^{(2)} n_{2}, K^{(3)} n_{3}\right)
$$

donde $\left(P_{C r}\right) n_{1} n_{2} n_{3}$ es una variable aleatoria discreta asociada a la variable $P_{c r}$.

Dicha variable se obtiene a partir de la resolución de los problemas (2.13) para cada

$$
i=1, ., n_{1} ; j=1, . ., n_{2} ; k=1, ., n_{3}
$$

tal y como se indicó en el apartado 3 de [13].

\section{Comentarios}

Como puede verse, la aplicación de la técnica de perturbación requiere la resolución de una sucesión de problemas (2.4), (2.5), (2.6), (2.7), etc. cada uno de los cuales es diferente del anterior y en general más complejo. Por otro lado también resulta muy laboriosa la obtención de expresiones que permita estimar los primeros momentos de la variable aleatoria $\mathrm{P}_{\mathrm{cr}}$. Asimismo no es sencillo por este procedimiento obtener aproximaciones de la función de distribución de la carga crítica.

En general las dificultades anteriores quedan solventadas aplicando el método de discretización, pues en primer lugar los sucesivos problemas a resolver son todos del mismo tipo lo que permite utilizar un mismo método de resolución numérica de diferencias finitas (elementos finitos) para cada uno de los problemas (2.13). En segundo lugar, por este procedimiento es posible al menos teóricamente aproximar tanto como se desee y de manera sencilla los momentos de $\mathrm{P}_{c r}$, basta para ello aumentar el número de puntos de discretización $\left(n_{1}, n_{2}\right.$ y $\left.n_{3}\right)$. También resulta inmediato la construcción de funciones de distribución que aproximen a la de $P_{c r}$, aunque para este menester sea necesario aumentar considerablemente el número de puntos de discretización para que dicha aproximación sea aceptable.

A continuación se expone el caso de pieza con rigidez constante, para el cual la relación entre la carga crítica $P_{c r}(\omega)$ y las constantes de los vínculos elásticos $K_{1}(\omega), K_{2}(\omega)$ y $K_{3}(\omega)$ puede expresarse en términos de una ecuación trascendente estocástica.

\subsection{Pieza con rigidez constante $(E \mathrm{I}(\mathrm{x})=\mathrm{El})$}

Haciendo en (2.1) el cambio de variable independiente $\mathrm{x}=\mathrm{Lz}$, el problema queda reducido al intervalo $[0,1]$ y se transforma en:

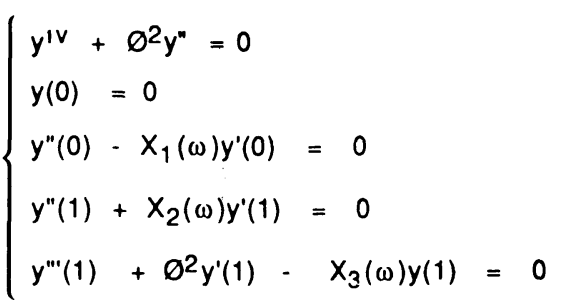

donde las derivadas son ahora respecto a la variable $z$, con

$$
\begin{aligned}
& \oslash^{2}=P \frac{L^{2}}{E I}, X_{1}(\omega)=K_{1}(\omega) \frac{L}{E I}, \\
& X_{3}(\omega)=K_{3}(\omega) \frac{L^{3}}{E I} \text { y } X_{2}(\omega)=K_{2}(\omega) \frac{L}{E I},
\end{aligned}
$$

donde $\oslash, X_{1}, X_{2}$ y $X_{3}$ son variables adimensionales.

La ecuación característica es $r^{4}+\emptyset^{2} r^{2}=0$ siendo sus raices $r=0$ (doble) y $r= \pm \varnothing_{i}$, de ahí que la solución general sea:

$y(x)=c_{1}+c_{2} z+c_{3} \operatorname{sen} \emptyset z+c_{4} \cos \oslash z$

Por otro lado, las condiciones de contorno dan lugar a un sistema homogéneo cuya matriz de coeficientes es:

$$
\left(\begin{array}{cccl}
1 & 0 & 0 & 1 \\
0 & -x_{1}(\omega) & -x_{1}(\omega) \emptyset & -\emptyset^{2} \\
0 & x_{2}(\omega) & \left(-\oslash^{2} \operatorname{sen} \emptyset+\right. & \left(-\oslash^{2} \cos \emptyset\right. \\
& & \left.+x_{2}(\omega) \varnothing \cos \emptyset\right) & \left.-x_{2}(\omega) \emptyset \operatorname{sen} \emptyset\right) \\
-x_{3}(\omega) & \emptyset^{2}-x_{3}(\omega) & -x_{3}(\omega) \operatorname{sen} \emptyset & -x_{3}(\omega) \cos \emptyset
\end{array}\right)
$$

Anulando el determinante de dicha matriz, después de varias simplificaciones se obtiene la siguiente ecuación trascendente estocástica:

$$
\begin{aligned}
& {\left[\left(x_{1}(\omega) x_{2}(\omega) x_{3}(\omega)-x_{2}(\omega) x_{3}(\omega)-x_{1}(\omega) x_{3}(\omega)\right)+\right.} \\
& \left.+\left(-x_{3}(\omega)-x_{1}(\omega) x_{2}(\omega)\right) \emptyset^{2}+\oslash^{4}\right] \oslash \text { sen }+ \\
& {\left[2 x_{1}(\omega) x_{2}(\omega) x_{3}(\omega)+\left(x_{2}(\omega) x_{3}(\omega)+x_{1}(\omega) x_{3}(\omega)\right) \oslash^{2+}\right.} \\
& \left.+\left(-x_{2}(\omega)-x_{1}(\omega)\right) \oslash^{4}\right] \cos \oslash-2 x_{1}(\omega) x_{2}(\omega) x_{3}(\omega)=0
\end{aligned}
$$


La carga crítica $P_{c r}(\omega)$ resulta ser:

$$
P_{c r}(\omega)=\frac{E I}{L^{2}} \emptyset_{1}{ }^{2}(\omega)
$$

donde $\phi_{1}(\omega)$ es la raíz positiva más pequeña de la ecuación anterior.

La versión determinista de la ecuación (2.17), es decir $\mathrm{X}_{1}, \mathrm{X}_{2}, \mathrm{X}_{3}=$ cte, generaliza a la dada por Brush y Almroth en [5] para el caso de dos empotramientos elásticos.

A continuación se desarrolla un ejemplo en el cual se aplica el método de discretización a la ecuación estocástica (2.17), determinándose varias aproximaciones para los primeros momentos de la carga crítica.

\section{Ejemplo}

Sea una estructura como la que se indica en la figura 2.2 en la cual la pieza $A B$ sometida a pandeo está unida rígidamente en sus extremos a las piezas $A A^{\prime}$ y $B B^{\prime}$ articuladas en $A^{\prime}$ y $B^{\prime}$ respectivamente, asimismo la pieza B'B" paralela a AB está empotrada en su extremo B".

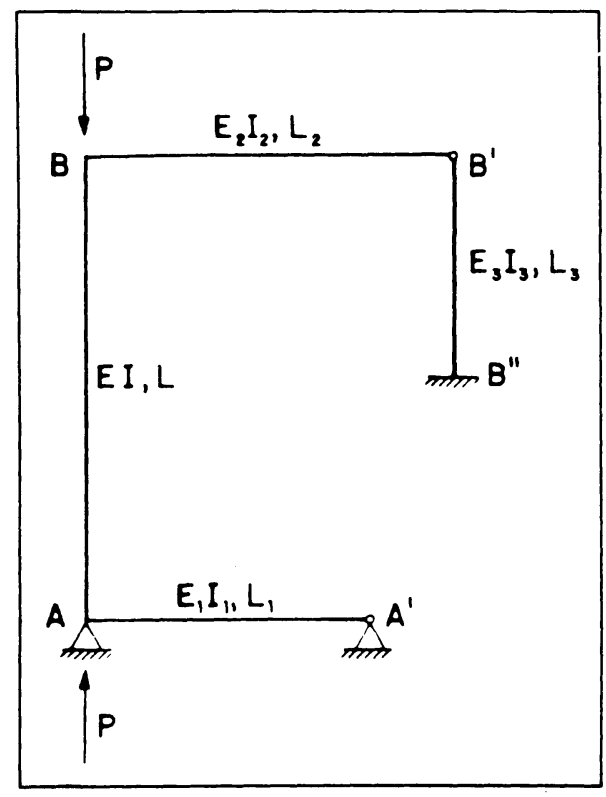

Fig. 2.2

Aislando ahora la pieza AB (v. Fig. 2.3) las constantes de los vínculos elásticos son respectivamente:

en $A$

$$
K_{1}=3 E_{1} l_{1} / L_{1}
$$

en $B$

$$
K_{2}=3 E_{2} I_{2} / L_{2}, K_{3}=3 E_{3} I_{3} / L_{3}{ }^{3}
$$

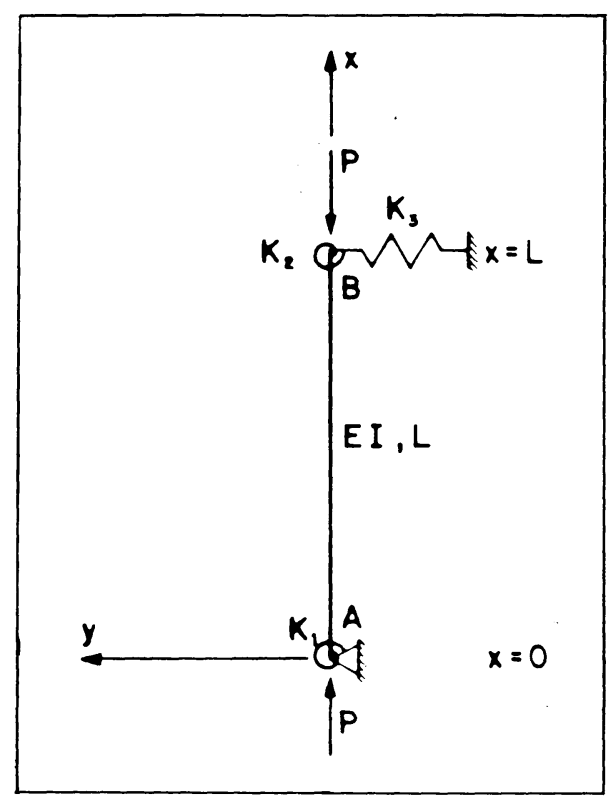

Fig. 2.3

de esta forma las constantes adimensionales $X_{1}, X_{2} y$ $\mathrm{X}_{3}$ del problema (2.14) son:

$$
\begin{aligned}
x_{1}=3 & \frac{E_{1} I_{1} / L_{1}}{E I / L}, \quad x_{2}=3 \frac{E_{2} I_{2} / L_{2}}{E I / L}, \quad x_{3}= \\
& =3 \frac{E_{3} I_{3} / L_{3}{ }^{3}}{E I / L^{3}},
\end{aligned}
$$

Estas expresiones permiten dar a su vez una idea del orden de magnitud de dichas constantes adimensionales en términos relativos de las rigideces y las longitudes de las piezas AA', BB' y B'B" respecto de las correspondientes de $\mathrm{AB}$.

Pasamos ahora a considerar el carácter ẩleatorio de $X_{1}, X_{2}$ y $X_{3}$ debido a la incertidumbre en el conocimiento preciso de las rigideces $E_{i} l_{i} y / 0$ las longitudes $L_{i} ; i$ $=1,2,3$ (El y $L$ deterministas).

Supongamos para este ejemplo que $L_{i}=L ; i=1,2,3$ y que las rigideces $E_{i} l_{i}$ son variables aleatorias uniformes con las siguientes distribuciones (análogamente, podrían considerarse otras distribuciones, tales como, la Normal, Gamma, Beta, Histogramas, etc.):

\section{Caso 1.9)}

$\mathrm{E}_{1} \mathrm{I}_{1}$ sobre el intervalo [1.0 El, 1.2 El], $\mathrm{E}_{2} \mathrm{I}_{2}$ sobre [1.5 El, $1.8 \mathrm{El}$ y $\mathrm{E}_{3} \mathrm{I}_{3}$ sobre [4.0 El, $4.8 \mathrm{El}$. De esta forma $\mathrm{X}_{i}(\omega)$, $i=1,2,3$ son también uniformes y en los siguientes intervalos:

$$
X_{1} \text { en }[3.0,3.6], X_{2} \text { en }[4.5,5.4] \text { y } X_{3} \text { en }[12.0,14.4]
$$




\section{(aso 2.)}

$E_{1} l_{1}$ y $E_{2} l_{2}$ sobre el intervalo [0.0 El, 0.2 El] y $E_{3} l_{3}=$ $\infty$ (determinista). Asi $X_{1}$ y $X_{2}$ son uniformes sobre [0.0, $0.6] \operatorname{con} X_{3}=\infty$ (apoyo rígido en $B$ )

\section{Caso 3.)}

$E_{1} I_{1}$ y $E_{2} I_{2}$ sobre el intervalo [3.0 El, $5.0 \mathrm{El}$ ] y $E_{3} I_{3}=$ $\infty$ (determinista). Las distribuciones de $X_{1}$ y $X_{2}$ son por tanto uniformes sobre $[9.0,15.0]$ con $X_{3}=\infty$.

Considerando ahora para todos estos casos un factor $\mathrm{EI} / \mathrm{L}^{2}=1000(\mathrm{~kg})$. La carga crítica es $\mathrm{P}_{\mathrm{cr}}(\omega)=1000 \phi_{1}$ ${ }^{2}(\omega)$ donde $\phi_{1}(\omega)$ es la raíz positiva más pequeña de la ecuación (2.17).

Resolviendo para cada uno de los casos la ecuación trascendente estocástica (2.17) mediante la técnica de discretización de las variables aleatorias, se han obtenido las siguientes aproximaciones $\beta^{*}{ }_{k}(n)=E$ $\left[\left(P_{c r}\right)^{k n, n, n] ~ d e ~ l o s ~ m o m e n t o s ~ d e ~ l a ~ v a r i a b l e ~ a l e a t o r i a ~}\right.$ $P_{c r}$, donde $n$ representa el número de puntos en los que se discretizan cada una de las variables aleatorias $\mathrm{X}_{1}, \mathrm{X}_{2}$ y $\mathrm{X}^{3}$.

En las Tablas 2.1, 2.2 y 2.3 se dan los respectivos valores con doble precisión para $n=2,4$ y 10 y cada uno de los casos $\left.1 .^{\circ}\right), 2^{\circ}$ ) y $3^{\circ}$ ).

TABLA 2.1

\begin{tabular}{|c|c|c|}
\hline \multicolumn{3}{|c|}{$x_{1}:\{3.0,3.6\}, x_{2}:\{4.5,5.4\}, x_{3}:(12.0,14.4\}$} \\
\hline \multicolumn{3}{|c|}{$x_{1}, x_{2}, x_{3}$ discretizadas en $n=2$ puntos } \\
\hline \multicolumn{3}{|c|}{ Momentos aproximados de $P_{c r}$ : } \\
\hline Media $=\beta^{\circ},(2)$ & $=0.1708268212787834 \times 10^{5}$ & $(\mathrm{~kg})$ \\
\hline Varianza $=\beta_{2}{ }_{2}(2) \cdot \beta^{2}{ }_{1}(2)$ & $=0.4019501304721832 \times 10^{6}$ & $\left(\mathrm{~kg}^{2}\right)$ \\
\hline Mom. ord. $3=\beta_{3}{ }_{3}(2)$ & $=0.5005635224206773 \times 10^{13}$ & $\left(\mathrm{~kg}^{3}\right)$ \\
\hline Mom. ord. $4=\beta^{*}{ }_{4}(2)$ & $=0.858618125991 \subseteq 091 \times 10^{17}$ & $\left(\mathrm{~kg}^{4}\right)$ \\
\hline \multicolumn{3}{|c|}{$x_{1}, x_{2}, x_{3}$ discretizadas en $n=4$ puntos } \\
\hline \multicolumn{3}{|c|}{ Momentos aproximados de $P_{c r}$ : } \\
\hline Media $=\beta^{\circ},(4)$ & $=0.1708261318849750 \times 10^{5}$ & $(\mathrm{~kg})$ \\
\hline Varianza $=\beta_{2} \cdot(4) \cdot \beta^{2}:(4)$ & $=0.4015218769773245 \times 10^{6}$ & $\left(\mathrm{~kg}^{2}\right)$ \\
\hline Mom. ord. $3=\beta_{3}{ }^{(4)}$ & $=0.5005549035008199 \times 10^{13}$ & $\left(\mathrm{~kg}^{3}\right)$ \\
\hline Mom. ord $4=\beta^{\circ}{ }_{4}(4)$ & $=0.8585954596983557 \times 10^{17}$ & $\left(\mathrm{~kg}^{4}\right)$ \\
\hline \multicolumn{3}{|c|}{$x_{1}, x_{2}, x_{3}$ discrelizadas en $n=10$ puntos } \\
\hline \multicolumn{3}{|c|}{ Momentos aproximados de $\mathrm{P}_{\mathrm{cr}}$ : } \\
\hline Media $=\beta_{1}^{*}(10)$ & $=0.1708261318011052 \times 10^{5}$ & $(\mathrm{~kg})$ \\
\hline Varianza $=\beta_{2}{ }_{2}(10) \cdot \beta_{1}^{2}(10)$ & $=0.4015217964837551 \times 10^{6}$ & $\left(\mathrm{~kg}^{2}\right)$ \\
\hline Mom. ord. $3=\beta_{3}{ }_{3}(10)$ & $=0.5005549022962373 \times 10^{13}$ & $\left(\mathrm{~kg}^{3}\right)$ \\
\hline Mom. ord. $4=\mathrm{B}_{4}{ }_{4}(10)$ & $=0.8585954561851566 \times 10^{17}$ & $\left(\mathrm{~kg}^{4}\right)$ \\
\hline
\end{tabular}

TABLA 2.2

\begin{tabular}{|c|c|c|}
\hline$x_{1}:[0.0 .0 .6\}$ & 1. $x_{3}=\infty$ & \\
\hline \multicolumn{3}{|c|}{$x_{1}, x_{2}$ discretizadas en $n=2$ puntos } \\
\hline \multicolumn{3}{|c|}{ Momentos aproximados de $P_{c r}$ : } \\
\hline Media $=\beta^{*}{ }_{1}(2)$ & $=0.1101713325002454 \times 10^{5}$ & $(\mathrm{~kg})$ \\
\hline Varianza $=\beta \cdot{ }_{2}\left(2, \cdot \beta \cdot{ }_{1}^{2}\right.$ (2) & $=0.2127045994710103 \times 10^{5}$ & $\left(\mathrm{~kg}^{2}\right)$ \\
\hline Mom. ord. $3=\beta_{3}, 21$ & $=0.1344262681642629 \times 10^{.3}$ & $\left(\mathrm{~kg}^{3}\right)$ \\
\hline Mom. ord. $4=\beta_{4}{ }_{4} \mid \mathrm{z}$ : & $=0.1488757759971831 \times 10^{\circ}$ & $\left(\mathrm{kg} \mathrm{g}^{4}\right.$ \\
\hline \multicolumn{3}{|c|}{$\mathrm{x}_{1}, \mathrm{x}_{2}$ discrelizadas $\in \mathrm{n} n=4$ puntos } \\
\hline \multicolumn{3}{|c|}{ Momentos aproximados de $P_{\text {cr: }}$} \\
\hline Media $=\beta^{*},(4)$ & $=0.1101712967775486 \times 10^{5}$ & $(k g)$ \\
\hline Varianza $=\beta \cdot{ }_{2}(4) \cdot \beta \cdot{ }^{2}(4)$ & $=0.2129912190465368 \times 10^{6}$ & $\left(\mathrm{~kg}^{2}\right)$ \\
\hline Mom. ord. $3=\beta_{3} \cdot(4)$ & $=0.1344266565141832 \times 10^{13}$ & $\left(\mathrm{~kg}^{3}\right)$ \\
\hline Mom. ord. $4=\beta^{*}{ }_{4}(4)$ & $=0.1488759648656973 \times 10^{: 7}$ & $\left(\mathrm{~kg}^{4}\right)$ \\
\hline \multicolumn{3}{|c|}{$x_{1}, x_{2}$ discretzzadas en $n=10$ puntos } \\
\hline \multicolumn{3}{|c|}{ Momentos aproximados de $P_{c r}$ : } \\
\hline Media $=\beta \cdot,(10)$ & $=0.1101712967775469 \times 10^{5}$ & $(\mathrm{~kg})$ \\
\hline Varianza $=\beta \cdot{ }_{2}(10) \cdot \beta \cdot{ }_{1}^{2}(10)$ & $=0.2129912190383933 \times 10^{6}$ & $\left(\mathrm{~kg}^{2}\right)$ \\
\hline Mom. ord $3=\beta_{3}{ }_{3}(10)$ & $=0.1344266565139714 \times 10^{13}$ & $\left(\mathrm{~kg}^{3}\right)$ \\
\hline Mom. ord $4=\beta_{4}{ }_{4}(10)$ & $=0.1488759648657361 \times 10^{17}$ & $\left(\mathrm{~kg} \mathrm{~g}^{4}\right)$ \\
\hline
\end{tabular}

TABLA 2.3

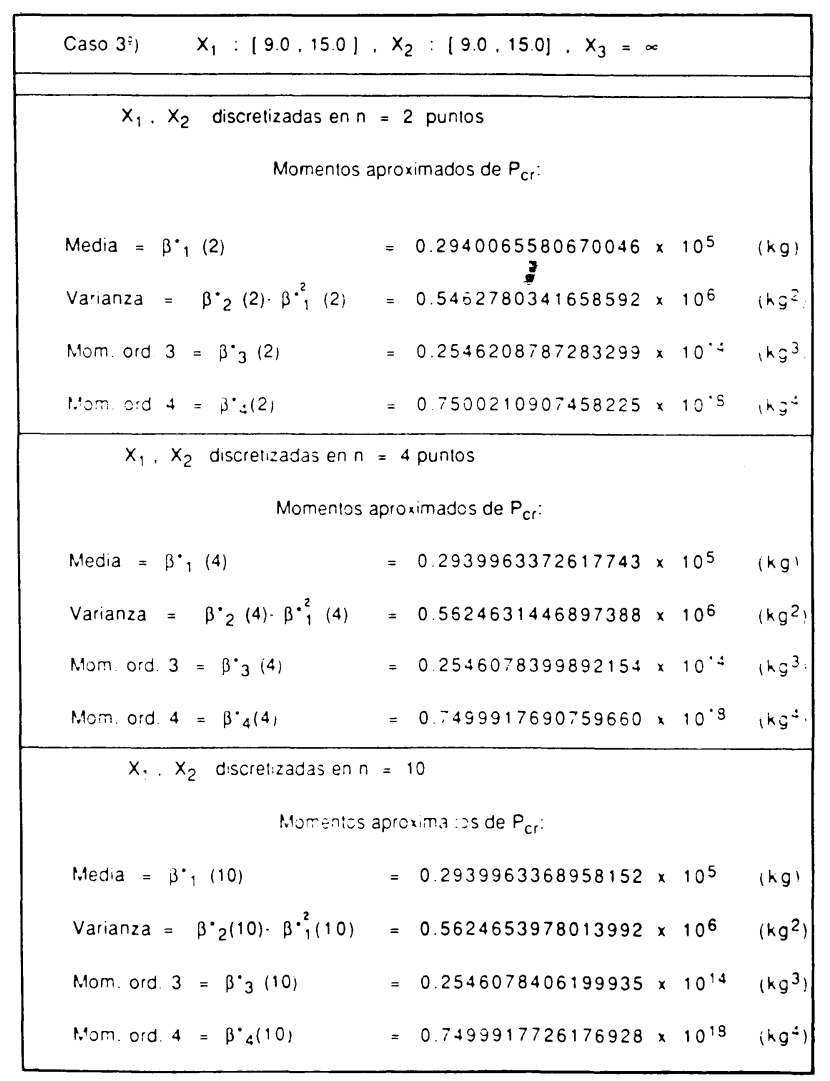


De los resultados obtenidos se deduce que en estos casos y para propósitos prácticos, basta con la aproximación obtenida tomando $n=2$, lo que equivale en definitiva a la resolución de ocho ecuaciones deterministas en $1^{\circ}$ ) y cuatro en $2^{\circ}$ ) y $3^{\circ}$ ) resultantes de discretizar en (2.17) cada una de las variables aleatorias en dos puntos.

Con objeto de valorar en alguna medida la influencia de la aleatoriedad de las condiciones de vínculo (para las distribuciones consideradas) en la aleatoriedad carga de pandeo utilizamos el coeficiente de variación:

$V\left[P_{c r}\right]=\left(D\left[P_{c r}\right] / E\left[P_{c r}\right) \times 100\right.$

Los valores obtenidos se dan en la Tabla 2.4:

TABLA 2.4

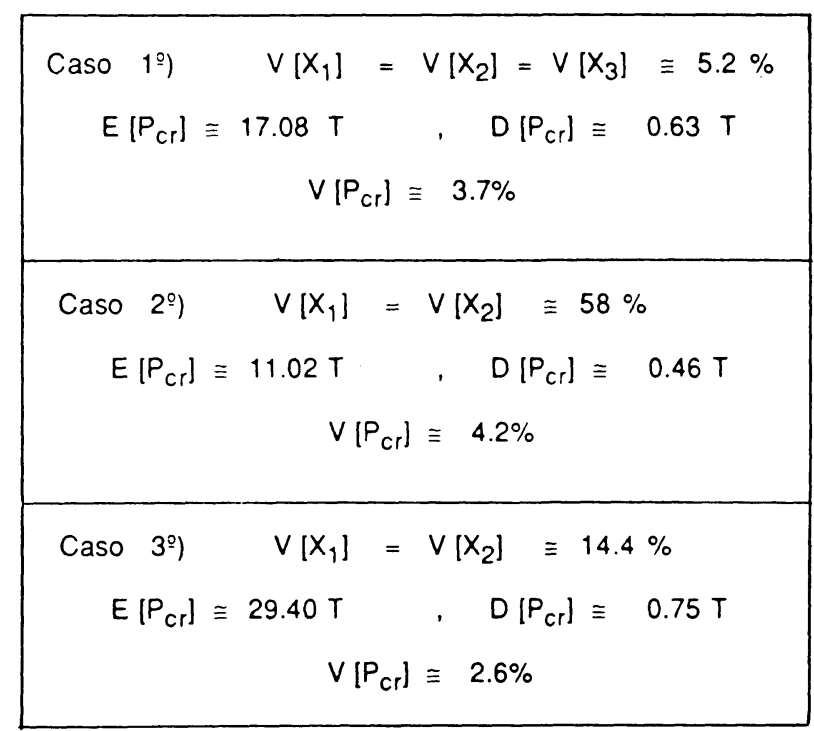

Estos resultados indican que el coeficiente de variación de $P_{c r}$ es en estos casos del mismo orden, sin embargo, la influencia de la aleatoriedad de las condiciones de vínculo en la de la carga de pandeo es para los casos 2.) y 3.) proporcionalmente menor que para el caso $1 . \%$.).

Como puede verse el método de discretización permite en forma sencilla obtener aproximaciones a los momentos de $\mathrm{P}_{\mathrm{cr}}$. Asimismo, pueden obtenerse también aproximaciones a la función de distribución, aunque para este menester es necesario, como ya se ha comentado, discretizar las variables $X_{i}$ en mayor número de puntos.

\section{APLICACIÓN A OTROS PROBLEMAS DE PANDEO ESTOCÁSTICO}

A continuación se exponen posibles aplicaciones del método de discretización a problemas de pandeo es(C) Consejo Superior de Investigaciones Científicas

Licencia Creative Commons 3.0 España (by-nc) tocástico tales como los de rigidez estocástica e imperfección inicial estocástica.

En cuanto al caso de rigidez estocástica consideremos la situación particular del problema (1.1) cuando la pieza tiene extremos articulados; en dicho caso las condiciones de contorno son deterministas y el problema se reduce a:

$$
\left\{\begin{array}{l}
\left(E I(x, \omega) y^{\prime \prime}\right)^{\prime \prime}+P y^{\prime \prime}=0 \\
y(0)=y^{\prime \prime}(0)=y(L)=y^{\prime \prime}(L)=0 \\
x \in[0, L]
\end{array}\right.
$$

Este problema es tratado por Scheidt y Purkert en [15] considerando que la pieza tiene sección circular con radio estocástico definido por el proceso $r(x, \omega)$, de forma tal que para $\mathrm{E}$ constante, $\mathrm{El}(\mathrm{X}, \omega)=\pi \mathrm{Er}^{4}(\mathrm{X}, \omega) / 4$ sea un proceso estocástico estacionario en sentido amplio (v. de la Rosa y Blázquez [14]). Asimismo dichos autores mediante la aplicación del método de Ritz transforman (3.1) en un problema de autovalores estocásticos matricial, obteniendo al mismo tiempo distribuciones límites de la carga de pandeo para diferentes parámetros del proceso $\mathrm{El}(\mathrm{x}, \omega)$.

Por nuestra parte indicamos la aplicación del método de discretización a aquellos casos en los cuales el proceso $\mathrm{El}(\mathrm{x}, \omega)$ pueda expresarse en función de un número reducido de variables aleatorias independientes $X_{i}$; $i=1, . ., q$.

Por ejemplo para el caso de una pieza de sección circular, $X_{i}$ puede representar el radio de la sección en distintos puntos $\xi_{\mathrm{i}} \operatorname{con} 0 \leq \xi_{1}<\xi_{2}<\ldots<\xi_{\mathrm{i}}<\ldots<\xi_{\mathrm{q}} \leq$ $L$ situados a lo largo de la pieza, con la hipótesis adicional de que la variación del radio entre zodos puntos consecutivos sea por ejemplo lineal. También puede considerarse en forma análoga el caso de una pieza de sección rectangular.

En estos casos, (3.1) se puede expresar en la forma:

$$
\left\{\begin{array}{l}
E I\left(x, X_{1}(\omega), \ldots, X_{q}(\omega)\right) y^{\prime \prime}+P y=0 \\
y(0)=y(L)=0 \\
x \in[0, L]
\end{array}\right.
$$

Dicho problema una vez aplicada la técnica de discretización a $X_{1}, X_{2}, \ldots X_{q}$ puede resolverse numéricamente por diversos métodos, 10 que permite obtener aproximaciones a los momentos y a la función de distribución de $\mathrm{P}_{\mathrm{cr}}(\omega)$.

Finalmente pasamos a comentar el problema de imperfección inicial estocástica, el cual es probablemente http://informesdelaconstruccion.revistas.csic.es 
uno de los más estudiados, entre los de pandeo estocástico.

Considerando el caso particular de (1.2) en el cual la rigidez es constante, se puede poner haciendo $x=L z$, $\mathrm{K}^{2}=\mathrm{P} / \mathrm{EI}, \phi=\mathrm{KL}$

$$
\left\{\begin{array}{l}
y^{\prime \prime}+\varnothing^{2} y=-\varnothing^{2} y^{*}(z, \omega) \\
y(0)=y(1)=0
\end{array}\right.
$$

La función de Green para este problema particular es:

$$
G(z, v)=\left\{\begin{aligned}
G_{1}(z, v)= & \frac{k}{\operatorname{sen} k L}[\operatorname{senkLz} \operatorname{senkL}(1-v)] \\
G_{2}(z, v) & =\frac{k}{\operatorname{sen} k L}[\operatorname{senkL}(1-z) \text { senkL }]
\end{aligned}\right.
$$

para $v<z<1$, luego la deformación adicional es:

$$
y(z, \omega)=\int_{0}^{1} G(z, v) \quad y^{*}(v, \omega) d v
$$

Boyce [2] y Jacquot [9] obtienen tomando esperanzas a partir de (3.5) teniendo en cuenta que la función de Green es determinista:

$$
\begin{aligned}
& E[y(z, \omega)]=\int_{0}^{1} G(z, v) E\left[y^{*}(v, \omega)\right] d v \\
& E\left[y^{2}(z, \omega)\right]= \\
& =\int_{0}^{1} \int_{0}^{1} G\left(z, v_{1}\right) G\left(z, v_{2}\right) E\left[y^{*}\left(v_{1}, \omega\right) y^{*}\left(v_{2}, \omega\right)\right] d v_{1} d v_{2}= \\
& =\int_{0}^{1} \int_{0}^{1} G\left(z, v_{1}\right) G\left(z, v_{2}\right) K\left(v_{1}, v_{2}\right) d v_{1} d v_{2}
\end{aligned}
$$

donde $\mathrm{K}\left(\mathrm{v}_{1}, \mathrm{v}_{2}\right)=\mathrm{E}\left[\mathrm{y}^{*}\left(\mathrm{v}_{1}, \omega\right) \mathrm{y}^{*}\left(\mathrm{v}_{2}, \omega\right)\right]$ es la función de autocorrelación del proceso $\mathrm{y}^{*}(z, \omega)$ (v. de la Rosa y Blázquez [14]).

Boyce en [2] considera que $y^{*}(z, \omega)$ es un proceso estacionario de media nula, así $\mathrm{E}[\mathrm{y}(\mathrm{z}, \omega)]=\mathrm{E}\left[\mathrm{y}^{*}(\mathrm{z}, \omega)\right]=0$ $V z \in[0,1]$ y $K\left(v_{1}, v_{2}\right)=R\left(v_{1}-v_{2}\right)$ donde $R$ es sólo función de la diferencia $\tau=\mathrm{v}_{1} \mathrm{v}_{2}$ siendo la varianza:

$$
\begin{aligned}
& D^{2}[y(z, \omega)]= \\
& =\int_{0}^{1} \int_{0}^{1} G\left(z, v_{1}\right) G\left(z, v_{2}\right) \cdot R\left(v_{1}-v_{2}\right) d v_{1} d v_{2}
\end{aligned}
$$

Dicho autor firma asimismo basándose en el teorema central del límite que en el supuesto de que $y^{*}(z, \omega)$ sea además débilmente correlado esto es:

$$
R\left(v_{1}-v_{2}\right)=\left\{\begin{array}{cc}
\rho\left(v_{1}-v_{2}\right) & \text { para }\left|v_{1}-v_{2}\right| \leq \varepsilon \\
0 & \text { para }\left|v_{1}-v_{2}\right|>\varepsilon
\end{array}\right.
$$

la distribución de $y(z, \omega)$ para cada $z \in[0,1]$ es aproximadamente Normal quedando por tanto definida a partir de (3.8).

Por su parte Jacquot [9] comenta que la suposición de estacionariedad para la imperfección inicial $\mathrm{y}^{\star}(z, \omega)$ es excesivamente fuerte ya que $y^{\star}(O, \omega)=y^{*}(L, \omega)=0$ con probabilidad uno, de ahí que proponga para la imperfección inicial un proceso tal cuya autocorrelación $\mathrm{K}$ $\left(v_{1}, v_{2}\right)$ sea una función que se anule en la frontera del cuadrado de vértices $(0,0),(0,1),(1,0)$ y $(1,1)$. En concreto sugiere expresiones de la forma:

$$
K\left(v_{1}, v_{2}\right)=\sum_{i=1}^{\infty} \sum_{j=1}^{\infty} K_{i j} \text { sen } i \pi v_{1} \quad \operatorname{senj} \pi v_{2}
$$

De esta manera:

$D^{2}[y(z, w)]=$

$=\sum_{i=1}^{\infty} \sum_{j=1}^{\infty} K_{i j} \int_{0}^{1} \int_{0}^{1} G\left(z, v_{1}\right) G\left(z, v_{2}\right) \operatorname{sen} i \pi v_{1} \operatorname{senj} \pi v_{2} d v_{1} d v_{2}$

Dicho autor expone para un caso particular en el que $\mathrm{K}\left(\mathrm{v}_{1}, \mathrm{~V}_{2}\right)=\sigma^{2} \operatorname{sen} \pi \mathrm{v}_{1} \operatorname{sen} \pi \mathrm{v}_{2}$ la relación existente entre el parámetro $P$ y la varianza de $y(z, \omega)$ para cada $z \epsilon$ $[0,1]$, poniendo de manifiesto que dicha varianza tiende a infinito cuando $P$ se acerca a $P_{c r}=\pi^{2} E I / L^{2}$.

La aplicación del método de discretización que proponemos para este caso de imperfecçión en la forma se basa en considerar dicha imperfección $\mathrm{y}^{*}(\mathrm{x}, \omega)$ como un desarrollo limitado de Fourier con coeficientes aleatorios:

$$
y^{*}(z, \omega)=\sum_{i=1}^{q} x_{i}(\omega) \operatorname{sen} \frac{i \pi x}{L}
$$

donde $X_{i} ; i=1, . ., q$ (con q pequeño) son variables aleatorias independientes.

Así (1.2) se reduce a:

$$
\left\{\begin{array}{l}
E l(x) y^{\prime \prime}+P y=-P \sum_{i=1}^{q} x_{i}(\omega) \text { sen } \frac{i \pi x}{L} \\
y(0)=y(L)=0
\end{array}\right.
$$

siendo inmediata la aplicación del método. 
Cuando $\mathrm{El}(\mathrm{x})=\mathrm{El}$ (cte) el desplazamiento adicional $y(x, \omega)$ puede expresarse en términos explícitos de $X_{i}$; $i=1, \ldots q$ pues:

$$
y(x, \omega)=\sum_{i=1}^{q} \frac{P X_{i}(\omega)}{P_{i}-P} \text { sen } \frac{i \pi x}{L}
$$

con

$$
P_{i}=\frac{i^{2} \pi^{2} E I}{L^{2}} ; i=1 \ldots q .
$$

Asimismo se destaca también que el caso de pieza cargada excentricamente (v. Fig. 3.1) con excéntricidades $e_{1}(\omega)$ y $e_{2}(\omega)$ aleatorias, puede reducirse a uno de imperfección inicial en la forma.

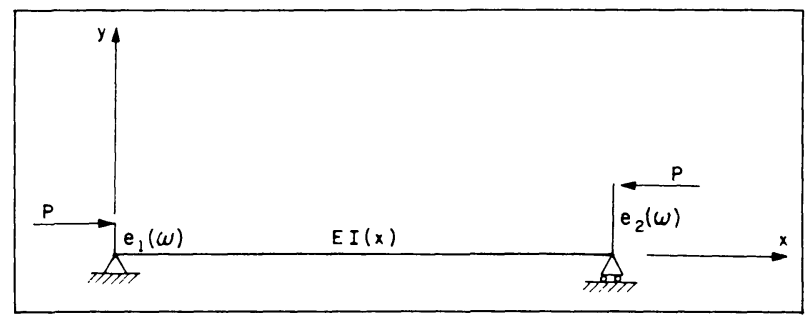

Fig. 3.1.

En efecto: Sea $\varphi(x)$ el polinomio de grado menor o igual a tres tal que $\varphi(0)=\varphi(L)=0, \varphi^{\prime \prime}(0)=\mathrm{P} \mathrm{e}_{1}(\omega) / E(0)$, $\varphi^{\prime \prime}(L)=P e_{2}(\omega) / E I(L)$, esto es:

$$
\varphi(x)=A+B(x-L)+C(x-L)^{2}+D(x-L)^{3}
$$

donde

$$
\begin{aligned}
& A=0, \quad B=\left(\frac{P e_{1}(\omega)}{6 E I(0)}+\frac{P e_{2}(\omega)}{3 E I(L)}\right) L \\
& C=P e_{2}(\omega) /(2 E I(L)), \\
& D=\left(\frac{P e_{2}(\omega)}{E I(L)}-\frac{P e_{1}(\omega)}{E I(0)}\right) /(6 L)
\end{aligned}
$$

Realizando ahora el cambio de variable

$$
y(x)=Z(x)+\varphi(x)
$$

queda:

$$
\left(E I(x) z^{\prime \prime}\right) "+P z^{\prime \prime}=-P\left(\varphi(x)+\frac{E l(x)}{P} \varphi^{\prime \prime}(x)\right)^{\prime \prime}
$$

junto con las siguientes condiciones de contorno homogéneas

(c) Consejo Superior de Investigaciones Científicas Licencia Creative Commons 3.0 España (by-nc)
$z(0)=z(L)=z^{\prime \prime}(0)=z^{\prime \prime}(L)=0$

Obsérvese que en el caso de pieza con rigidez El constante la ecuación queda en la forma:

$E I z^{I V}+P z^{\prime \prime}=-P \varphi^{\prime \prime}(x)$

equivalente a la (1.2). Esto indica que en lo referente a la determinación de la elástica de equilibrio (para $P$ distinto de autovalor), este problema de cargas aplicadas excéntricamente puede considerarse equivalente a otro de una pieza biarticulada con una imperfección inicial estocástica dada por $\mathrm{y}^{\star}(\mathrm{x})=\varphi(\mathrm{x})$, donde el desplazamiento adicional es $z(x)$.

Por otro lado hay que indicar que la equivalencia no es total, pues aquí la ley de momentos flectores es $M(x)=$ $=E I\left(z "(x)+\varphi{ }^{\prime \prime}(x)\right)$ mientras que en un caso de imperfección inicial $M(x)=E \mid z^{\prime \prime}(x)$ donde $z(x)$ representa ahora la desviación adicional.

\section{COMENTARIOS Y CONCLUSIONES}

El tratamiento de los problemas de pandeo desde un enfoque clásico o determinista y con teorías de segundo orden, ha demostrado ser una aproximación suficiente para la mayoría de los casos que se presentan en la práctica de la construcción. Sin embargo, la modelización estocástica de estos problemas y dentro también de las teorias de segundo orden, permite introducir de forma natural el concepto de fiabilidad, a partir de la determinación de la probabilidad de ruina o colapso, lo que no es posible desde los planteamientos deterministas. Por otra parte, no se ocultan las dificultades que surgen desde el nuevo enfogue, asi: 1.a) Elección adecuada de las variables aleatorias y procesos estocásticos que definen la geometría de la pieza y las características de los materiales, 2.) Problemas matemáticos a resolver más complejos.

En relación con la primera dificultad, debe observarse que la elección que se haga para la descripción estocástica, condicionará de alguna forma los resultados posteriores. En este sentido, algunos analistas realizan la elección en base a simplificar el tratamiento matemático del problema, lo cual, como destaca Jacquot [9], concretamente en relación con el problema de imperfección en la forma, puede limitar notablemente la validez de los resultados. En cuanto a la segunda, sería deseable disponer de la herramienta matemática adecuada para tratar eficazmente el problema, independientemente de las distribuciones que se tomen para las variables y procesos estocásticos inmersos en el mismo, objetivo, por otra parte, dificilmente alcanzable. 
Por lo que se refiere al trabajo desarrollado, el mismo se ha centrado fundamentalmente en el estudio de la influencia de la aleatoriedad de las condiciones de vínculo de una pieza recta sometida a pandeo, en la carga de pandeo. Dicho análisis es de gran utilidad para las aplicaciones, sobre todo en los casos donde la presencia inevitable de pequeños defectos de ejecución sea significativamente importante en las condiciones de vínculo.

En cuanto a la técnica de resolución, se han destacado las ventajas del método de discretización, esto es, su carácter sistemático y su exactitud. Asimismo, para el caso concreto de una pieza con rigidez constante, se han determinado mediante la aplicación del citado método, aproximaciones de los primeros momentos de la carga de pandeo para una elección arbitraria de las distribuciones de probabilidad que representan las constantes de los vínculos elásticos. También se ha indicado cómo puede aplicarse dicho método en la resolución de problemas tales como los de rigidez estocástica e imperfección inicial estocástica.

Finalmente se puede concluir que las ideas y método aplicado pueden extenderse con pocos cambios a situaciones distintas o más complejas que las aquí expuestas. En este sentido casos como por ejemplo el tratado por Roorda [11] sobre pandeo de una pieza con imperfección inicial estocástica y carga aplicada excéntricamente con excentricidades aleatorias, resultan de cómoda resolución empleando el método de discretización.

\section{REFERENCIAS}

1. AMAZIGO, J.C. (1969) "Buckling under axial compression of long cilindrical shells with random axisymmetric imperfections". Quartely of Applied Mathematics, Vol. XXVI, No. 4, pp. 557-566.

2. BOYCE, W.E. (1961) "Buckling of a colum with random initial displacements". Journal of the Aerospace Sciences, Vol. 28, No. 4, pp. 308-320.

3. BERNARD, M. C. and BOGDANOFF, F.L. (1971) "Buckling of columns with random initial displacements". Journal of the Engineering Mechanics Division, Proc. ASCE 97 (EM 3), pp. 755-771.
4. BUDIANSKY, and B. HUTCHINSON, J.W. (1966) "A survey of some buckling problems". AIAA Journal, Vol. 4, No. 9, pp. 1505-1510.

5 .BRUSH, D.O. and ALMROTH, B.O. (1975) "Buckling of Bars, Plates and Shells". Mc Graw-Hill, Inc., New York.

6. FRASER, W.B. (1965) "Buckling of a structure with random imperfections". Ph. D. Thesis, Harvard University.

7. FRASER, W.B. and BUDIANSKY, B. (1969) "The buckling of a column with random initial deflections". Jornal of Applied Mechanics, Vol. 36, pp. 233-240.

8. HAINES, C.W. (1965) "An analysis of stochastic eigenvalue problems". Ph. D. Thesis, Rensselaer Polytechnic Institute, Troy, New York.

9. JACQUOT, R.G. (1972) "Nonstationary random column buckling problem". J. Engr. Mechs, Civ., Proc. ASCE 98 (EMS), pp. 1173-1182.

10. PERRY, S.H. (1966) "Statistical variation of buckling strength". Ph. D. Thesis, University College, London.

11. ROORDA, J. (1975) "The random nature of column failure" J. Struct. Mech., 3(3) pp. 239-257.

12. ROMERO, J.L. (1989) "Tratamiento numérico de algunos problemas estocásticos. Contribución al estudio del pandeo estocástico". Tesis Doctoral, Universidad Politécnica de Madrid.

13. ROMERO, J.L. y ROSA, E. de la. (En prensa) "Resolución de algunos problẹmas estocásticos mediante discretización". Informes de la Construcción (C.S.I.C.), Madrid.

14. ROSA, E. de la y BLÁZQUEZ, R. (1985) "Procesos Estocásticos. Vibraciones Aleatorias". E.T.S.I.C.C. y P. Madrid.

15. SCHEIDT, J. and PURKERT, W. (1983) "Random Eigenvalue Problems". North Holland, New YorkAmsterdam Oxford.

16. TIMOSHENKO, S.P. and GERE, J.M. (1961) "Theory of Elastic Stability". 2 nd edition. Mc Graw-Hill, New York. 